\title{
Finding safety in medical education
}

\section{P Stevens}

\section{The doctor-patient and teacher-learner relationships remain at the core of the rapidly changing practice of medicine. Medical education must embrace a safety culture if these relationships are to serve patients well.}

\begin{abstract}
"What gives value to travel is fear. It is the fact that, at a certain moment, when we are so far from our own country. . . we are seized by a vague fear, and an instinctive desire to go back to the protection of old habits." Albert Camus
\end{abstract}

The doctor-patient relationship has been at the core of medicine for centuries. However, the last decade of the 20th century has seen radical accelerating change in the context in which that relationship is embedded. It is increasingly complex and hurried ${ }^{2}$ and, disturbingly, it is fraught with substantial risk to the patient.

Similarly, the teacher-learner relationship exists at the core of medical education, which must wrestle with the same accelerating change. While the focus on patient safety gains increasing attention in the clinical setting, it is slow to gain strategic awareness among medical educators. Were it of biological origin, the discovery of an epidemic that results in 44 000-98 000 deaths annually in the US alone $^{3}$ would quickly find its way to the formal medical curriculum, for changes in biology and technology (particularly of such magnitude) are readily incorporated by medical faculties. Patient safety pushes medical education into unfamiliar territory. Complex systems, ${ }^{4}$ culture, and teamwork ${ }^{6}$ are not mainstream topics in the traditional curriculum. So, where do we start?

In this issue of QSHC Aron and Headrick $^{7}$ set out an excellent proposal by offering a systems metaphor for discerning safety in medical education. They argue persuasively that the "organizational defences" of the medical education system fail, and the result is inadequate education for doctors to provide safe care. Students and, importantly, their future patients are at risk. The authors focus strategically on important elements of medical education that include entrance requirements, curriculum, organizational culture, assessment, and accreditation. The list is daunting but on target and highly strategic. All the same, the strategy might benefit from further reflection on deep seated trends that envelop medical practice and, inevitably, contemporary medical graduates.

\section{THE EVOLVING DOCTOR-PATIENT AND TEACHER-LEARNER RELATIONSHIPS}

Consider the relentless transformation that occurs in two essential bonds in health care and medical education - the doctor-patient and the teacher-learner relationships.

The traditional doctor-patient relationship is frequently amended by the imperative for doctors to collaborate more effectively with each other and with other health professionals. What has traditionally been a "one to one" bond for the patient now may require effective integration with a "one to many" relationship. Another element that has dramatically altered the interaction between doctors and patients is the Internet. There was a time when medical knowledge was a principal source of authority for the doctor. ${ }^{8}$ Now both the patient and doctor have access to the same information. The doctor is now responsible for integrating and customizing information for the benefit of the patient. While professional authority still plays an important role in how the doctor provides counsel, the ubiquity of information brings about a substantial realignment. There exists little formal education for dealing with these inevitable modifications of the doctorpatient relationship.

The teacher-learner relationship is also evolving. Leach ${ }^{9}$ suggests that the studies of Hubert and Stuart Dreyfus provide a valuable insight into how doctors learn. Working in the 1970s, the Dreyfus brothers studied how pilots acquire knowledge and skills. ${ }^{10}$ They described five progressive stages in the continuum of learning: novice, advanced beginner, competent, proficient, and expert. The novice learns by careful application of a defined set of rules. The advanced beginner demonstrates greater skill by applying those rules to new unforeseen situations. As learners acquire expertise, learning sheds rule bound behavior and becomes more intuitive. The Dreyfus model can be readily applied to the continuum of medical education. The process begins when medical students move from novice to advanced beginner during their medical school experiences. Trainees in graduate medical education progress to acquire competence, and expertise resides with the senior teaching faculty.

The emergence of new knowledge, however, drives constant and dynamic reorientation of the teacher-learner relationship along the continuum. In the traditional teacher-learner relationship, senior (expert) doctors impart knowledge to (novice and advanced beginner) students and (increasingly competent) graduate trainees. That having been said, most would concede that expertise in information technology currently resides with the student and trainee, while the senior doctor is the novice. When it comes to patient safety, all learners along the continuum from medical student to teaching faculty are novices. In medicine, rapidly accumulating new knowledge increasingly merges the traditional roles of teacher and learner.

\section{IMPORTANCE OF A "SAFETY CULTURE" IN MEDICAL EDUCATION}

By addressing the importance of establishing a "safety culture" in medical education, Aron and Headrick ${ }^{7}$ have focused on a fundamental and strategic issue. In this regard there is much to learn from the study of cultural realignment in other complex high risk organizations. For example, in their efforts to discern organizational models for safe systems Weick and Sutcliffe ${ }^{11}$ have investigated the culture of so called "high reliability organizations" (HROs) such as US Navy aircraft carriers and nuclear power plants, and have extended their observations to describe lessons for healthcare systems.

It may be informative to extrapolate such lessons one step further to examples in systems for medical education. Weick and Sutcliffe ${ }^{11}$ point out that HROs adopt a culture that centers on mindfulness and constant attention to failures. An example for medical education might be morbidity and mortality conferences that relentlessly explore the root causes of failure in recent care events. HROs readily adapt organizational structure temporarily to meet unusual situations-for example, the student who teaches the teacher how to use the most recent computer software for electronic order entry. Finally, HROs are constantly mindful of the unexpected and exploit such events for organizational learning-for example, timely bedside teaching and learning that focus in depth (perhaps even celebrate as a learning opportunity) a trainee's near miss in the care of a patient.

Weick and Sutcliffe ${ }^{11}$ refer to reliability as a "dynamic non-event". Expressed another way, when adverse events are prevented, nothing happens. An educational tradition that places greater 
emphasis on disease treatment than on illness prevention is slow to reward doctors and students when the "dynamic non-event" of patient safety happens.

Davidoff has suggested that one additional cultural barrier to improvement in the healthcare system is shame because " . . . improvement means that, however good your performance has been, it is not as good as it could be". ${ }^{5}$ By extension, educators who have devoted their careers to educational systems that were historically successful, but now are insufficient, must embrace the need for valid improvement if knowledge for safety is to find its way to their students.

\section{"Improving health and health care begins with the focus on improving medical education"}

Finally, effective and safe clinical systems require leaders who are relentlessly committed to safety and reliability. ${ }^{41}$ This requirement may necessitate new criteria for educational institutions in the recruitment and promotion of their organizational leadership.
Aron and Headrick serve patients well with their proposal that medical educators should radically rethink systems for preparing future doctors. ${ }^{7}$ Improving health and health care begins with the focus on improving medical education. Strategic improvement-based on adoption of a systems approach, reflection on the realigned doctor-patient and teacherlearner relationships, transformed culture, and strong leadership—provides the appropriate start. The pace of change in medicine and health care insists on a measure of urgency. Patients rightfully trust the profession to educate doctors to incorporate such change into their care. Finding safety in medical education can provide reassuring confirmation of that trust.

Qual Saf Health Care 2002;11:109-1 10

Correspondence to: Dr D P Stevens, Vice President, Medical School Standards and Assessment, Association of American Medical Colleges, 2450 N Street NW, Washington, DC 20037, USA; dstevens@aamc.org

\section{REFERENCES}

1 Camus A. Notebooks, 1935-1942. In:

Palmer P. The courage to teach. San Francisco: Jossey-Bass, 1998: 39.

2 Ludmerer KM. Time to heal: American medical education from the turn of the Century to the era of managed care. New York: Oxford University Press, 1999.

3 Institute of Medicine. To err is human: building a safer health care system. Washington, DC: National Academy Press, 1999

4 Mohr JJ, Batalden PB. Improving safety on the front lines: the role of clinical microsystems. Qual Saf Health Care 2002;11:45-50.

5 Davidoff F. Shame: the elephant in the room. Qual Saf Health Care 2002;11:2-3.

6 Risser DT, Simon R, Rice MM. A structured teamwork system to reduce clinical errors. Error reduction in health care. San Francisco: Jossey-Bass, 1999: 235

7 Aron DC, Headrick LA. Educating physicians prepared to improve care and safety is no accident: it requires a systematic approach. Qual Saf Health Care 2002;11:168-73.

8 Starr P. The social transformation of American medicine. New York: Basic Books, 1982.

9 Leach DC. Competence is a habit. JAMA 2002;287:243-4.

10 Dreyfus SE, Dreyfus HL. A five stage model of the mental activities involved in directed skills acquisition. Unpublished manuscript supported by US Air Force Office of Scientific Research under contract F49620-79-0063 with the University of California, Berkeley.

11 Weick KE, Sutcliffe KM. Managing the unexpected. San Francisco: Jossey-Bass, 2001.

\section{Quality improvement research: understanding the science of change in health care}

\section{R Grol, R Baker, F Moss}

\section{Essential for all who want to improve health care.}

E xpectations of healthcare services are ever increasing and those delivering care no longer hold the monopoly of opinion on what constitutes good or best care. To earn the label "good enough", care must meet standards expected by consumers as well those of expert providers. Headlines in newspapers, statements in policy documents, and many analyses, surveys and reports repeatedly highlight serious problems in healthcare delivery related to underuse, overuse, or misuse of care. ${ }^{1}$ Health systems are sometimes unsafe and frequently we harm patients who have trusted us with their care. There is an endemic failure to engage patients with decisions about their care. We know there are problems; we just need to change so that care can be made safer and better.

Everyone-authorities, policy makers, and professionals-seems to accept the need for change. New initiatives aiming to cure our ailing systems come in droves. This is an international phenomenon. Many initiatives are linked to programmes that capture a particular approach-for example, evidence based medicine; accreditation and (external) accountability; total quality management; professional development and revalidation; risk management and error prevention; organisational development and leadership enhancement; disease management and managed care; complex adaptive systems; and patient empowerment. They may differ in perspective. Some focus on changing professionals, others on changing organisations or interactions between parts of the system; some emphasise self-regulation, others external control and incentives; some advocate "bottom up" and others "top down" methods. Despite their differences, however, each aims to contribute to better patient care-and they might, but the evidence for understanding their likely impact is not robust and many seem based more on belief than rigorous research of value, efficacy, or feasibility. ${ }^{2}$ From what we know, no quality improvement programme is superior and real sustainable improvement might require implementation of some aspects of several approaches-perhaps together, perhaps consecutively. We just do not know which to use, when to use them, or what to expect.

More evidence and understanding is required. At least 40 good systematic reviews and numerous controlled trials are available, ${ }^{34}$ but many of the trials can be criticised because, for example, randomisation or analysis was conducted at the patient level while the intervention focused on professionals or teams, and outcome parameters are often poorly chosen or are difficult to compare. Most studies were conducted in the USA, limiting generalisations to other systems. Some strategies are better studied than others. We know more 
about CME, audit and feedback, reminders and computerised decision support than about organisational, economic, administrative and patient mediated interventions. New methods including the effects of problem based education or portfolio learning, TQM, breakthrough projects, risk management methods, business process redesign, leadership enhancement, or sharing decisions with patients are not well studied. Studying the effects of specific strategies in controlled trials will provide some answers to some questions about effective change, but will not address some of the basic questions about the critical success factors in change processes. They need to be complemented by observational and qualitative studies.

Health care is becoming increasingly complex and the problems are large. It is unrealistic to expect that one specific approach can solve everything. A qualitative study by Solberg et al of critical factors supporting implementation of change showed that a mixture of professional and organisational factors is crucial. "Give attention to many different factors and use multiple strategies" is the message. ${ }^{6}$ Although we may know that multifaceted strategies combining different actions and measures linked to specific obstacles to change are usually more successful than single interventions, ${ }^{7}$ we know little about which components of such complex interventions are effective in different target groups. So, while there is some general knowledge, there is little detailed understanding of the "black box" of change.

We need to learn about change in the real world of health care and the crucial determinants of successful improvement. New thinking about healthcare settings as complex adaptive systems emphasises the importance of experimenting with multiple approaches and discovering what works best. ${ }^{8}$ Small changes can sometimes have large effects-but we have little understanding about which small changes to use in which settings and their likely impact.

For real change and sustained improvement a tailored research methodology is essential. The full range of methodology has yet to be established, but will include contributions from epidemiology, behavioural sciences, educational research, organisational and management studies, economics, and statistics (box 1). Theoretical models of evaluations of complex interventions propose a phased approach (theoretical phase, definition of the components of
Box 1 Some research approaches for quality

improvement research

- Observational studies of existing change processes

- In-depth qualitative studies on critical success factors and barriers to change improvement programmes

- Systematic reviews of both the impact of different strategies and the influence of specific factors on change

- Well designed cluster randomised trials

- Systematic sampling and interpretation of experiences of change

- Methods for developing valid and sensitive indicators for measuring change

- Meta-analyses of large samples of improvement projects

- Methods for evaluation of large scale implementation and change programmes

- Economic analyses of resources needed for effective change and improvement of care

- Statistical process control

the intervention, small scale explanatory trial, followed by larger trials and research into long term implementation). Clearly, different research methods are required for different phases, ${ }^{9}$ but it is essential that, despite the eclectic base of the research, researchers from different faculties and disciplines come together to collaborate in this complex field and that the vogue for "quick fixes" is replaced with sustained research.

To stimulate and support debate about research on quality improvement and change management in health care we have commissioned a series of papers to provide an overview of some relevant methodologies. The first two papers are published in this issue and more will follow. Pope et al ${ }^{10}$ explore some of the qualitative methods that can be used to gather information about the delivery of good quality care, and Wensing and Elwyn $^{11}$ consider some of the key issues related to measurement of patients views. Forthcoming issues of QSHC will include papers that describe research methods for indicator development in primary care; a methodology for evaluating small scale improvement projects; methods for evaluating quality improvement programmes; research designs for randomised controlled trials in quality improvement; and economic evaluations of change management.
There is a recognised process for the development of new drugs, their introduction into routine practice, and their establishment in the treatment of defined conditions. As knowledge about a drug is accrued, new and better patterns of treatment gradually become established. Similar measured approaches are needed to help develop and establish better, safer systems of care. "Change management" is a discipline central to health care. The academic base that supports change management and quality improvement in health care should underpin all clinical and managerial learning programmes. The science of change management is not new, but there is a long way to go before we will understand enough to be able to transform care so that it is "good enough" to meet everyone's expectations of quality and safety.

Qual Saf Health Care 2002;11:110-111

\section{Authors' affiliations}

R Baker, R Grol, Guest Editors, Quality Improvement Research Series

F Moss, Editor in Chief, Quality and Safety in Health Care

Correspondence to: Professor R Grol, Centre for Research on Quality in Health Care (WOK), PO Box 9101, 6500 HB Niimegen, The Netherlands; R.Grol@hsv.kun.nl

\section{REFERENCES}

1 Bodenheimer T. The American health care system. The movement for improved quality in health care. N Engl J Med 1999;340:48892.

2 Grol R. Beliefs and evidence in changing clinical practice. BM 1997:315:418-21.

3 Grol R. Improving the quality of medical care Building bridges among professional price, payer profit, and patient satisfaction. JAMA 2001;286:2578-85.

4 Grimshaw JM, Shirran L, Thomas R, et al. Changing provider behavior: an overview of systematic reviews of interventions. Med Care 2001;39(8 Suppl 2): II2-45

5 Solberg L, Brekke M, Fasio J, et al. Lessons from experienced guideline implementers: attend to many factors and use multiple strategies. Jt Comm J Qual Improv 2000;26:171-88.

6 Solberg L. Guideline implementations: what the literature doesn't tell us. Jt Comm J Qual Improv 2000;26:525-37.

7 Wensing $M$, van der Weijden T, Grol R. Implementing guidelines and innovations in general practice: which interventions are effective? Br J Gen Pract 1998;48:991-7.

8 Plsek PE, Greenhalgh T. The challenge of compexity in health care. BM 2001;323:625-8.

9 Campbell M, Fitzpatrick R, Haines A, et al Framework for design and evaluation of complex interventions to improve health. $B M$ 2000;321:694-6.

10 Pope C, van Royen P, Baker R. Qualitative methods in research on healthcare quality. Qual Saf Health Care 2002;11:148-52.

11 Wensing M, Elwyn G. Research on patients' views on the evaluation and improvement of quality of care. Qual Saf Health Care 2002;11:153-7. 
Patient safety

\section{Championing patient safety: going global}

\section{Sir Liam Donaldson}

A resolution on patient safety being considered by the World Health Assembly will move patient safety on to the world health stage.

$\mathrm{T}$ he early days of any programme to improve the quality of health care are always the most challenging, but potentially the most exciting. Raising awareness of the issues, inspiring front line staff to rise to the challenges, influencing policy makers to make a commitment and invest resources, persuading a health service to accord it a priority, reassuring the doubters, picking out the leaders-these are the challenges. And so it has been with patient safety. The problem of medical error had been present since organised clinical practice began, yet no one recognised it as a fundamental concept. Airline safety had been systematically improved over three decades by understanding and strengthening systems, yet few people were struck by the parallels with health care. Lives were being lost and people being made ill daily in hospitals around the world, yet no pattern was recognised. Over a period of 5 years spanning the end of the 20th century and the beginning of the 21 st, this position has been transformed.

Influential individual thinkers ${ }^{1-3}$ and major governmental reports ${ }^{4-6}$ have quantified the problem of medical error and its impact on patients, have described the context of risk and unsafe systems, and have scoped the action necessary to produce change.

This year patient safety has begun to move from in-country programmes and some between-country collaborations ${ }^{7}$ on to the world health stage. In January 2002 the Executive Board of the World Health Organisation $(\mathrm{WHO})^{8}$ passed a resolution put forward by the governments of the UK, Japan, Belgium, the Islamic Republic of Iran, and Italy calling on the WHO to establish a programme on patient safety. The Executive Board is the executive committee of the WHO, consisting of one representative from each of 32 of its 191 member states. It sits twice a year with the Director General of the WHO and her staff in attendance to discuss policy and progress on major global health matters. Selectively, and with careful deliberation, it identifies important new areas of work and makes recommendations to the World Health Assembly.

The resolution on patient safety set out four proposed areas for action:

- Determination of global norms, standards and guidelines for the definition, measurement and reporting of adverse events and near misses in health care and the provision of support to countries in developing reporting systems, taking preventive action, and implementing measures to reduce risks.

- Promotion of framing of evidencebased policies including global standards that will improve patient care, with particular emphasis on such aspects as product safety, safe clinical practice in compliance with appropriate guidelines and safe use of medicinal products and medical devices, and creation of a culture of safety within healthcare organisations.

- Development of mechanisms, through accreditation and other means, to recognise the characteristics of healthcare providers that offer a benchmark for excellence in patient safety internationally.

- Encouragement of research into patient safety.

In May 2002 the World Health Assembly will consider the resolution and, if accepted, the drive for safer health care will be a worldwide endeavour, seeking to bring benefits to patients in countries rich and poor, developed and developing, in all corners of the globe.

Qual Saf Health Care 2002;11:112

Correspondence to: Sir Liam Donaldson, Chief Medical Officer, Department of Health, Room 111 , Richmond House, 79 Whitehall, London SWIA 2NS, UK

Liam.Donaldson@doh.gsi.gov.uk

\section{REFERENCES}

1 Leape L. A systems analysis approach to medical error. J Eval Clin Pract 1997;3:213-22.

2 Reason J. Human error. Cambridge: Cambridge University Press, 1990.

3 Berwick DM, Leape LL. Reducing errors in medicine. Qual Health Care 1999;8:145-6.

4 Kohn LT, Corrigan JM, Donaldson MS, eds. To err is human: building a safer health system. Washington, DC: National Academy Press, 2000.

5 Department of Health. An organisation with a memory. Report of an Expert Group on Learning from Adverse Events in the NHS. London: The Stationery Office, 2000

6 Department of Health. Building a safer NHS for patients: implementing 'An Organisation with a Memory'. London: Department of Health, 2001.

7 US Department of Health and Human Services Press Release. US and UK sign agreements to collaborate on health care quality and fighting bioterrorism, 10 October 2001 (www.hhs.gov/news)

8 World Health Organisation. World Health Organisation Executive Board Resolution EB 109.R 16, 18 January 2002 (www.who.int/gb/EB_WHA/E/E_docEB 109.htm 


\section{Patient safety research: does it have legs?}

\section{R J Lilford}

\section{Patient safety research: where does it fit in?}

R esearch into patient safety is highly topical. The Agency for Health Care Research and Quality spends about $£ 40 \mathrm{M}$ per year under this heading and the UK has established a Patient Safety Research Programme which I direct. Patient safety research is somewhat unusual in that it works back from effect to cause; while most research asks about the effects of structures and process on outcomes, patient safety research starts with the outcome-iatrogenic injuryand asks how it might be avoided. A research programme with an emphasis on safety is needed to determine how and why safety is undermined and hence to develop and evaluate practices targeting safety as their main objective. However, many other research programmes concerned with improving quality generally will impact on safety and a dialogue with these programmes is essential. Similarly, managerial organisations with special responsibility for safety have come into being in many countries. Such organisations, which focus specifically on safety (such as the English National Patient Safety Authority (NPSA)), need to mesh with other organisations (such as the Commission for Health Improvement) responsible for quality generally. Patient safety can be seen as a kind of knowledge management, continually learning, educating and motivating. Patient safety programmes (whether research or managerial) have to be highly connected to the organisations they seek to influence and require a deep understanding, not only of scientific matters, but of the policy environment in which they work.

Patient safety agencies and research programmes have a special duty to reduce single acts which have serious consequences. Note that although the disaster can be traced directly to a single act, that act itself will have multiple antecedent "causes". This, then, is where patient safety interventions get their bite; they intervene in the chain of events where the probability of the untoward event is the product of the probabilities of (independent) antecedent events. This leads us into consideration of the forms that research into patient safety might take.
Patient safety research has a role in:

- Identifying the nature, extent, and context of iatrogenic injury (including errors of omission)

- Uncovering the factors antecedent to injury, especially the underlying behavioural causes

- Developing and evaluating interventions designed to reduce error

All of these involve a wide range of research methodologies.

\section{IDENTIFYING THE NATURE, EFFECT, AND CONTEXT OF IATROGENIC INJURIES}

Enumerating and categorising error can be done by counting reports (reporting systems, litigation records) or by investigating all cases of opportunity for error in an attempt to ascertain both numerator and denominator information and hence measure incidence. Yesterday's research project can be today's routine data system, and many countries have established standing mechanisms to solicit, record, and act on reports of untoward incidents. Such systems go beyond traditional reporting procedures for drug reactions, device failures, transfusion reactions, falls from bed, and needle stick injuries. The English programme has commissioned research into factors (especially cultural factors) that may affect willingness to report error (www.publichealth.bham.ac.uk/psrp).

However, such denominator free data underestimate many errors. This is important when management action is predicated, not just on the existence of problems, but on their incidence. Thus, it does not matter in terms of policy if drug calculation errors have been underestimated - there are far too many anyway and we need to act. But deciding whether to divert national resources to improve "pain to needle times" for patients with a heart attack or to reduce delay in operating on fractured neck of femur would require more accurate measurement of the scale of each problem. Unbiased measurement of error is also needed for comparative purposesfor instance, when monitoring the performance of healthcare providers or studying the effects of action to improve safety. Aggregated statistics are notoriously unreliable. ${ }^{12}$ Review of case notes is a widely used method for measuring error rates. However, it is subject to a number of identified biases ${ }^{34}$ and the problem that sicker patients have more opportunity for error. Enhanced technologies are being developed to measure error, such as digitally imaging endoscopic surgery and installation of cameras in operating theatre lights. The definition and unbiased measurement of error will be discussed at a forthcoming Anglo-American conference on methodological issues in patient safety research.

\section{DISCOVERING UNDERLYING CAUSES}

Deeper understanding of the causes of error builds on extensive work in other industries such as air traffic control, the nuclear industry, and others. Evolutionary selection did not equip the human mind for the complex technologies which it went on to create, so we are now prey to a disturbing range of psychological inadequacies. ${ }^{5}$ High risk industries reduce this problem by automation and close coupled systems. However, the continued presence of the human operator is required for those functions not easily automated and to intervene when events move outside system parameters. This latter, particularly, is a task for which human cognition is supremely ill suited. In these highly automated environments, where error is rare but catastrophic, the human operator is the "intelligent knowledge base" in the system, yet it is precisely this knowledge based problem solving which fails under stressful conditions leading, for example, to an incident at a nuclear plant in Ohio. ${ }^{6}$ On the other hand, well practised routine procedures which have become intuitive can also fail, for example, if the operator's attention is distracted-a factor identified as causal in some $6.5 \%$ of surveyed incidents in nuclear power plants. ${ }^{7}$ Nor do all errors originate at the operator level: the literature is littered with examples of failures attributable to organisational and cultural factorsdespite two similar incidents, management at Three Mile Island nuclear power plant had done nothing to prevent its recurrence ${ }^{8}$; at Bhopal the plant superintendent was untrained for his job ${ }^{9}$; NASA top management cleared Challenger to launch because they were unaware of a launch constraint put in place by the NASA booster project manager ${ }^{10}$; the bosun of the Herald of Free Enterprise did not close the bow doors because "it wasn't part of his job", even though earlier he had relieved from duty the crewman responsible for doing $\mathrm{So}^{11}$; and so forth. Qualitative research has proved invaluable in helping to unravel the 
complex social dynamics which determine safety in health services, ${ }^{12}$ and behavioural interventions have reduced accident rates in many industries. ${ }^{13}$

\section{DEVELOPING AND EVALUATING INTERVENTIONS}

Basic research into the antecedents of injury should lead to development of interventions designed to reduce risk. The potential effects can be modelled from the epidemiology and the degree of confidence in the intervention. If the desired outcome is an almost inevitable consequence of an intervention, then agencies should simply act. For example, a number of recent deaths in England have followed the administration of undiluted potassium chloride. So, get the stuff off the wards and we will prevent these deaths. The effects of other interventions may be less certain and controlled before and after studies may be needed to provide really convincing evidence. In that case, a proof of principle study (analogous to a phase 1 drug trial) may be needed to refine the intervention. For example, I have put out a call for a study to determine the effects of various types of simulation and drill to improve the management of acute obstetric emergencies. A large trial randomising all the labour wards in the country must await the results of these initial studies.

In the end, patient safety will be enhanced by automating procedures that can be automated (e.g. interpretation of heart rate traces in labour ward/ automated dispensing of drugs), trapping errors before they occur (e.g. online reminders), reducing pitfalls at the interfaces between care settings (e.g. by linking hospital and community prescribing systems), improving the design of procedures and equipment (e.g. delivery systems which preclude inadvertent intrathecal administration of neurotoxic drugs), and education (e.g. simulations to teach procedures). Culture seems to be improved by introducing specific measures of this sort (which then have beneficial knock on effects) rather than by non-specific exhortation. ${ }^{13}{ }^{14}$ However, bringing about meaningful directed change requires resources and large scale managerial action. A large challenge for patient safety research is to work with managers to introduce change around an evaluation framework (preferably involving before and after measurements in both control and intervention sites). The role of patient safety research is to get the evidence about what is likely to work, then to proselytise for change based on that evidence and, above all, to encourage managers to innovate in such a way that the whole world may learn. However, we should be wary of inadvertently creating the problems we wish to avoid through an overzealous campaign: educational interventions designed to promote road safety awareness among school leavers have the consistent and apparently perverse results of increasing road deaths (mediated by the unexpected effect of prompting early acquisition of driving licences).

Managers and policy makers beware; the road to hell is paved with good intentions. So, promulgate plausible service delivery interventions, but first liaise with those who commission research so that epistemologically sound prospective evaluations can be built in from the start. The Patient Safety Research Programme in England will work closely with the NPSA and others to ensure this happens.

\section{ACKNOWLEDGEMENT}

The author thanks Rachel Anderson (Birmingham, UK) and Paul Barach (Chicago, USA) for helpful advice on earlier drafts.

Qual Saf Health Care 2002;11:113-114
Correspondence to: Professor R J Lilford, Director of the Patient Safety Research Programme, Department of Health, and Professor of Clinical Epidemiology, University of Birmingham, Edgbaston, Birmingham B15 2TT, UK; R.J.Lilford@bham.ac.uk

\section{REFERENCES}

1 Jencks SF, Daley J, Draper D, et al. Interpreting hospital mortality data: the role of clinical risk adjustment. JAMA

1988;260:3611-6.

2 Dubois RW, Rogers WH, Moxley JH, et al. Hospital inpatient mortality: is it a predictor of quality? N Engl J Med 1987;317:1674-80.

3 Hayward RA, Hofer TP. Estimating hospital deaths due to medical errors. JAMA 2001;286:415-20.

4 Neale G, Woloshynowych M, Vincent C. Exploring the causes of adverse events in NHS hospital practice. J R Soc Med 2001:94:322-30.

5 Reason J. Actions not as planned: the price of automation. In: Underwood G, Stevens R eds. A speech of consciousness. London: Academic Press, 1979

6 NUREG. Loss of Main and Auxilliary Feedwater Event at the Davis-Besse Plant on June 9, 1985. NUREG-1 154. Washington, DC:US Nuclear Regulatory Commission, 1985

7 Rasmussen J. What can be learned from human error reports? In: Duncan K, Gruneberg M, Wallis D, eds Changes in working life. London: Wiley, 1980.

8 Kemeny J. The need for change: the legacy of TMI. Report of the President's Commission on the Accident at Three Mile Island. New York: Pergamon, 1979

9 Morehouse W, Subramaniam MA. The Bhopal tragedy. New York: Council on
International and Public Affairs, 1986.

10 Report of the Presidential Commission on the Space Shuttle Challenger Accident. Washington DC: Government Printing Agency, 1986

11 Sheen, Mr Justice. MV Herald of Free Enterprise. Report of Court No. 8074 Formal Investigation. London: Department of Transport, 1987

12 Firth-Cozens J, Mowbray D. Leadership and the quality of care. Qual Health Care 2001:10(suppl III): 113-7.

13 Krause TR, Seymour KJ, Sloat KEM Long-term evaluation of a behaviour based method for improving safety performance: a meta-analysis of 73 interrupted time-series replications. Safety Sci 1999:32:1-18.

14 Nasanen M, Saari J. The effects of positive feedback on housekeeping and accidents at a shipyard. J Occup Accidents 1987:8:237-50 